\title{
Biometria de frutos e diásporos de Cryptocarya aschersoniana Mez e Cryptocarya moschata Nees (Lauraceae)
}

\author{
Pedro Luís Rodrigues de Moraes ${ }^{1,2}$ \\ Marcelo Corrêa Alves ${ }^{3}$
}

Biota Neotropica v2 (n1) http://www.biotaneotropica.org.br/v2n1/pt/abstract?article+BN01302012002

\author{
Recebido em 17 de janeiro de 2002 \\ Revisado em 26 de março de 2002 \\ Aceito em 29 de abril de 2002
}

\author{
Pedro Luís Rodrigues de Moraes Bolsa FAPESP (99/05004-5) de Pós Doutoramento \\ ${ }^{2}$ Laboratório de Melhoramento de Plantas, CENA-USP, Caixa Postal 96, 13400-970, Piracicaba, SP, Brasil; Autor para \\ correspondência: E-mail: plmoraes@cena.usp.br \\ ${ }^{3}$ Seção Técnica de Informática, ESALQ-USP, Caixa Postal 9, 13401-000, Piracicaba, SP, Brasil
}

\begin{abstract}
Summary
[Biometry of fruits and diaspores of Cryptocarya aschersoniana Mez and Cryptocarya moschata Nees (Lauraceae)]. Data of the biometry of fruits and diaspores of Cryptocary a aschersoniana Mez and C. moschata Nees are presented. Fruits were collected from 12 populations of seven different localities of southeast Brazil. Using the logistic regression and the linear discriminant analysis, the species were classified according to the equations generated by these techniques. For entire fruits, the variables length, diameter, surface and volume were selected and accounted for $94.4 \%$ of concordance among the associations of expected and observed probabilities. Similarly, the variables length, diameter, surface and volume of diaspores were selected accounting for $88.0 \%$ of concordance. Cluster analysis was also performed. Variances within and among plants were observed in fruits and diaspores of both species. All variables were important for discrimination of groups. The three groups obtained either for fruits or diaspores were related with a gradation in their dimensions. Distinct grouping indicates presence of genetic variability within collected material. Additionally, to determine whether there was occurrence of isometry, the allometric relationships between length and diameter in fruits and diaspores were examined. Using the major axis technique, the $\ln$ (diameter) on $\ln$ (length) for fruits and diaspores were regressed. Fruits of $C$. aschersoniana tended to show positive allometry, while those of $C$. moschata tended to present isometry. For diaspores, both species had a tendency towards positive allometry.
\end{abstract}

Keywords - Biometry, allometry, fruit size, fruit shape, major axis regression, cluster.

\section{Resumo}

[Biometria de frutos e diásporos de Cryptocarya aschersoniana Mez e Cryptocarya moschata Nees (Lauraceae)]. Apresentam-se os dados da biometria de frutos e diásporos de Cryptocarya aschersoniana Mez e C. moschata Nees (Lauraceae). Os frutos foram coletados de 12 populações de sete localidades diferentes do sudeste brasileiro. Utilizando-se a regressão logística e a análise de função linear discriminante, as espécies foram classificadas de acordo com as equações geradas por essas técnicas. Para os frutos, as variáveis comprimento, diâmetro, superfície e volume foram selecionadas e apresentaram 94,4\% de concordância entre as associações de probabilidades esperadas e observadas. Similarmente, as variáveis comprimento, diâmetro, superfície e volume de diásporos foram selecionadas e apresentaram $88,0 \%$ de concordância. Realizou-se análise de agrupamento. Observou-se variabilidade dentro e entre plantas nos frutos e nos diásporos de ambas as espécies. Todas as variáveis foram importantes na discriminação dos grupos. Os três grupos formados tanto para frutos como para diásporos basearam-se numa gradação de suas dimensões. A obtenção de grupos distintos indica presença de variabilidade genética no material coletado. Adicionalmente, examinaram-se as relações alométricas entre o comprimento e o diâmetro de frutos e diásporos para a determinação de ocorrência de isometria. Utilizou-se a técnica do "eixo maior" para a regressão do $\ln$ (diâmetro) pelo $\ln$ (comprimento) de frutos e diásporos. Os frutos de C. aschersoniana apresentaram tendência à alometria positiva, enquanto que os de C. moschata uma tendência à isometria. Para diásporos, ambas as espécies apresentaram tendência à alometria positiva.

Palavras-chave - Biometria, alometria, tamanho de frutos, forma de frutos, regressão de eixo maior, agrupamento 


\section{Introdução}

A família Lauraceae é pantropical possuindo cerca de 50 gêneros (Rohwer 1993a), tendo provavelmente um número de espécies entre 2.500 e 3.500 , distribuídas predominantemente na América tropical e subtropical, sudeste asiático, Oceania, Madagascar e algumas poucas espécies na África (Rohwer 1993b). Estima-se que o número de espécies neotropicais existentes esteja em torno de 700 a 800, pertencentes a 31 gêneros (van der Werff 1988, 1991). Cryptocarya é pantropical com cerca de 350 espécies, a maioria delas na Malásia (Rohwer 1993b), e cerca de 10 espécies na América do Sul (Kostermans 1937, Vattimo-Gil 1966). O nome Cryptocarya significa fruto escondido (do grego, kryptos - escondido e káryon fruto), devido ao completo envolvimento do fruto pelo eixo floral acrescente (Bernardi 1962, Vattimo-Gil 1966), principal caráter que distingue este gênero dos demais dentro da família.

Segundo Vattimo-Gil (1966), ocorreriam sete espécies do gênero na Mata Atlântica brasileira: $C$. aschersoniana Mez, C. granulata Vattimo-Gil, $C$. jacarepaguensis Vattimo-Gil, $C$. micrantha Meisn., $C$. minima Mez, C. moschata Nees e $C$. saligna Mez. Em vista da distribuição geográfica e abundância relativa dos indivíduos, aliados às dificuldades presentes na identificação dos espécimens, as espécies $C$. aschersoniana e $C$. moschata foram consideradas as de maior interesse para o desenvolvimento deste trabalho. Tem-se que $C$. aschersoniana ocorre do Rio Grande do Sul ao Espírito Santo, com presença também em Mato Grosso do Sul, Mato Grosso e Goiás; C. moschata distribui-se do Rio Grande do Sul até Pernambuco, predominantemente ao longo da Escarpa Atlântica (Coe-Teixeira 1965, Kostermans 1937, 1938, Vattimo-Gil 1966). As espécies $C$. aschersoniana e $C$. moschata apresentam áreas de sobreposição, principalmente no sul e sudeste, sendo que a primeira ocorre predominantemente nas áreas de mata mesófila semidecídua, enquanto que a segunda ocorre quase que exclusivamente ao longo da mata atlântica sensu stricto (Joly et al. 1999). O tamanho e a forma dos frutos são freqüentemente utilizados nas descrições das espécies brasileiras de Cryptocarya, podendo ser características discriminantes destas. Porém, estes caracteres geralmente não são avaliados por estudos de campo que descrevam os padrões morfológicos e morfométricos encontrados ao longo da distribuição geográfica, a partir de material fresco (Howard 1981). Na literatura, tem-se que as dimensões dos frutos das espécies estudadas, em sua grande maioria, são provenientes de exsicatas de herbário, sendo que a porção carnosa dos frutos, que é oriunda do receptáculo floral, encontra-se consideravelmente diminuída pelo processo de desidratação. Desta forma, C. aschersoniana apresenta frutos com um tamanho máximo de $3,0 \mathrm{~cm}$ de comprimento por $2 \mathrm{~cm}$ de diâmetro, enquanto $C$. moschata apresenta frutos com até $2,8 \mathrm{~cm}$ de comprimento por $2 \mathrm{~cm}$ de diâmetro, valores esses que não representam as médias e são obtidos da mensuração de um número reduzido de frutos desidratados, a partir de material de herbário (Mez
1889, Kostermans 1937, 1938, Vattimo-Gil 1966, 1979).

Este trabalho teve como objetivo investigar se as variáveis morfométricas de frutos e diásporos de Cryptocarya aschersoniana Mez e de C. moschata Nees são adequadas por si só para a discriminação das espécies estudadas, avaliando quantitativamente a variabilidade dentro e entre espécies.

\section{Material e Métodos}

O material utilizado no presente trabalho constou de frutos maduros das espécies Cryptocarya aschersoniana Mez e C. moschata Nees, coletados em diversas áreas do estado de São Paulo e sul de Minas Gerais (tabela 1).

A identificação das espécies foi feita a partir de material herborizado e depositado no herbário da Escola Superior de Agricultura "Luiz de Queiroz" (ESA), sob os números: Cryptocarya aschersoniana, ESA: 65.041, 65.042, 65.043, 65.044, 65.045, 65.046, 65.048, 65.049, 65.050, 65.051, 65.052, 65.054, 65.059, 65.061; Cryptocarya moschata, ESA: 5.721, 5.722, 5.723, 5.736, 6.942, 7.118, 7.139, 7.837.

A coleta dos frutos foi feita diretamente nas copas, ou sob as árvores marcadas, a partir de frutos caídos, evitando-se coletar aqueles que estivessem predados ou danificados, certificando-se de não serem oriundos de árvores vizinhas. Os frutos coletados foram acondicionados em sacos plásticos, devidamente etiquetados, e foram transportados para o laboratório.

No laboratório, foram medidos o comprimento e o diâmetro dos frutos inteiros através de um paquímetro. Os diásporos (pericarpo + semente) foram medidos após a remoção da parte pulposa, feita com o auxílio de um canivete, ou por simples pressão entre os dedos.

Para o cálculo da superfície e volume dos frutos e diásporos, utilizou-se a metodologia proposta por Turrel (1946), descrita em Moraes \& Alves (1997).

Metodologias para discriminação das espécies através da biometria de frutos e diásporos foram desenvolvidas através de análise discriminante (PROC DISCRIM, SAS Institute 1989) e regressão logística (PROC LOGISTIC, SAS Institute 1989). As análises foram feitas separadamente para frutos inteiros e diásporos porque, no momento da separação, não foi mantida a correspondência entre frutos e respectivos diásporos.

Foi aplicada a técnica stepwise (passo a passo) para seleção de variáveis que compuseram o modelo, e foi testada a existência de multicolinearidade entre as variáveis selecionadas (VIF, índice de condicionamento e tolerância).

Utilizando-se da técnica do "eixo maior" ou "eixo principal" (major axis ou principal axis; Jolicouer \& Heusner 1971, Sokal \& Rohlf 1995), calculou-se a regressão de Modelo II adotado em vista da existência de erros nas duas variáveis e inexistência de uma variável independente. A técnica aplicada nas variáveis diâmetro $v s$. comprimento de fruto e diâmetro vs. comprimento de diásporo permite testar a presença ou ausência de 
similaridade geométrica de frutos e diásporos, conforme discussão apresentada por Mazer \& Wheelwright (1993). A técnica permite testar a hipótese de nulidade de isometria (forma se mantém constante através da amplitude de tamanhos). A hipótese alternativa, por sua vez, nos dá indícios de alometria (LaBarbera 1989), onde são constatados indícios de que há modificação de forma em decorrência de variação dos tamanhos.

Quando o coeficiente da regressão se aproxima de 1, não há indícios de que se deva rejeitar a hipótese de nulidade, o que implica na aceitação da idéia de isometria. Valores diferentes de 1 dão indícios de que se deva rejeitar a hipótese de nulidade, o que conduz à idéia da existência de alometria.

Este tipo de regressão difere da regressão ordinária calculada pelo método de mínimos quadrados e há uma inversão da hipótese de nulidade testada através dela. Conclusões à respeito da proximidade do coeficiente a 1 se embasam no intervalo de confiança (5\%) calculado para o parâmetro estimado.

Adicionalmente, fez-se uma análise de agrupamento por árvores das duas espécies, a partir das médias das variáveis envolvidas para frutos inteiros e diásporos, pelo procedimento CLUSTER (SAS Institute 1989) que se baseia na distância Euclidiana. Selecionou-se o método de ligação média (average linkage) para realização do agrupamento.

Para Cryptocarya aschersoniana foram utilizados 1.487 frutos inteiros e 1.283 diásporos de 37 e 36 árvores, respectivamente (coletados em 2000). Para $C$. moschata, foram utilizados nas análises 1.892 frutos inteiros e 1.764 diásporos de 22 árvores (coletados em 1992).

\section{Resultados e Discussão}

A partir das medições básicas de comprimento e diâmetro de frutos e diásporos, foram calculadas as respectivas razões entre diâmetro e comprimento, bem como suas superfícies e volumes, com o intuito de melhor representar suas formas e tamanhos. Em média, as dimensões dos frutos inteiros de $C$. aschersoniana foram significativamente maiores que as de C. moschata. Por outro lado, as dimensões dos diásporos de ambas as espécies mostraram-se, em média, bastante semelhantes (tabela 2), não revelando uma descontinuidade marcante entre as espécies. Os resultados obtidos mostram que as distribuições se sobrepõem, para todas as variáveis, concordando com o fato de que ambas as espécies são bastante similares morfologicamente, o que faz com que a identificação das mesmas, a partir de material herborizado, possa tornar-se difícil. Isto pode ser constatado pela grande confusão encontrada nas identificações desses materiais nos herbários. No campo, as espécies também são confundidas quando ocorrem associadas no mesmo local, promovendo um tratamento similar de nomes populares, tais como: canela-batalha, batalha, batalheira, ou canelafogo. Adicionalmente, dados isoenzimáticos corroboram as evidências morfológicas e de anatomia de madeira
(Richter 1981) de que essas espécies são bastante próximas geneticamente (P.L.R. Moraes, dados não publicados).

As funções lineares discriminantes com as variáveis selecionadas resultaram em modelos cujas estimativas mais se associaram aos dados originais, quando comparados aos das estimativas obtidas pela análise de regressão logística e através da análise discriminante com todas as variáveis. $\mathrm{Na}$ análise discriminante com as variáveis comprimento, diâmetro, superfície e volume de frutos inteiros, obteve-se $94,4 \%$ de concordância entre as associações de probabilidades esperadas e observadas, com altos coeficientes de correlação (Somers' D = 0,889; Gamma $=0,890 ;$ Tau-a $=0,438 ; \mathrm{c}=0,945)$, e com porcentagens semelhantes entre a sensibilidade e a especificidade na tabela de classificação $(81,7 \%$ e $88,8 \%$, respectivamente). Da mesma forma, as variáveis comprimento, diâmetro, superfície e volume foram selecionadas para diásporos, com $88,0 \%$ de concordância, e também com altos coeficientes de correlação (Somers' D $=0,762 ;$ Gamma $=0,763 ;$ Tau-a =0,379; $\mathrm{c}=0,881$ ), e com maior porcentagem de especificidade do que a de sensibilidade na tabela de classificação $(84,8 \%$ e $63,2 \%$, respectivamente). Tudo isto nos dá indícios da boa associação entre as estimativas calculadas através da análise discriminante e a verdadeira espécie à qual pertenciam os frutos, dando crédito à ferramenta desenvolvida.

As variáveis razão diâmetro/comprimento, de frutos e de diásporos, foram excluídas do modelo em decorrência dos indícios de multicolinearidade detectados através do VIF (fator de inflação de variâncias e índices de condicionamento).

Desta forma, para frutos inteiros, a análise discriminante resultou nas seguintes funções lineares discriminantes:

C. aschersoniana $=-633,27+356,69($ comprimento $)+$ 607,83(diâmetro) - 41,42(superfície) - 41,16(volume)

C. moschata $=-616,99+355,31$ (comprimento $)+$ 598,55(diâmetro) - 40,29(superfície) - 42,96(volume)

Para diásporos, as equações foram:

C. aschersoniana $=-2.213+1.235$ (comprimento) + 3.079(diâmetro) - 176,64(superfície) - 462,18(volume)

C. moschata $=-2.255+1.254$ (comprimento) + 3.114(diâmetro) -183,65(superfície) - 456,87(volume)

Para se testar a validade da análise discriminante e sua adequação, submeteram-se os dados a um processo de reclassificação que indicou que $82,65 \%$ de frutos de $C$. aschersoniana foram classificados corretamente em $C$. aschersoniana. Para frutos de $C$. moschata, a classificação em C. moschata foi de $87,16 \%$. A partir do modelo obtido através das medidas de diásporos, observa-se que a classificação correta daqueles de $C$. aschersoniana foi de $61,50 \%$, sendo de 77,20\% para aqueles de C. moschata (tabela 3).

Com base nesses resultados apresentados pelas análises de regressão logística e de função linear discriminante, evidenciou-se a existência de uma grande homogeneidade dentro dos grupos formados pelas 
espécies, a partir dos dados das variáveis selecionadas, o que possibilitou uma classificação adequada, com uma alta porcentagem de concordância, para cada espécie. No entanto, em vista da utilização de variáveis quantitativas em que a variação pode ser influenciada sobremaneira por causas de variação não mensuráveis no campo, não se pode afirmar taxativamente que a biometria de frutos possa ser utilizada como ferramenta determinante da classificação das espécies estudadas. Sendo assim, não se descarta a utilização das tradicionais variáveis qualitativas para a classificação nos grupos taxonômicos. Entretanto é bastante promissora a possibilidade de se utilizar o método de classificação biométrico como uma análise preliminar em vista da facilidade e rapidez de aplicação.

Além de uma ferramenta preliminar, propõe-se a classificação biométrica como uma ferramenta auxiliar, sendo mais uma informação para os casos nos quais a classificação tradicional se mostre insuficiente ou gere uma classificação duvidosa. Por fim, embora não sendo absolutamente confiável, demonstra-se que a classificação baseada na biometria pode ser capaz de uma boa probabilidade de acertos, o que pode torná-la útil em situações em que não se disponham de condições para a aplicação do método tradicional, ou em que se possam tolerar as probabilidades de erro envolvidas no processo de classificação através da biometria, devidamente quantificadas através das estatísticas que avaliam os métodos de classificação.

A decisão em se utilizar variáveis quantitativas e a presente abordagem biométrica deveu-se pela impossibilidade de utilização de caracteres qualitativos da morfologia de frutos, tais como presença ou ausência de costulação no pericarpo do fruto, na distinção destas espécies. Moraes \& Paoli (1995) mostraram a inadequação deste caráter para a caracterização de indivíduos de $C$. moschata, por variar inclusive dentro de um mesmo indivíduo, que pode apresentar frutos lisos ou costulados. Da mesma forma, com a obtenção dos frutos de $C$. aschersoniana para o presente trabalho, foi possível observar este mesmo fato ocorrendo entre e dentro dos indivíduos das populações amostradas. Ainda, o caráter coloração da parte pulposa do fruto também mostrou-se altamente variável dentro e entre as espécies.

Sob o contexto da forma e tamanho dos frutos, para todas as árvores de $C$. aschersoniana, a linha de regressão do "eixo maior" foi: "In(diâmetro de fruto) = $0,2134+1,3078 \ln$ (comprimento de fruto)", com o coeficiente angular apresentando alometria positiva significativa (figura 1A). Em sete das 37 árvores de $C$. aschersoniana, o coeficiente angular calculado foi $<1$, sendo que em cinco casos este não foi significativamente $<1$. Com isto, apenas duas árvores apresentaram alometria negativa significativa. Das 30 árvores que apresentaram coeficiente angular $>1,13$ apresentaram alometria positiva significativa, uma vez que tiveram o limite inferior do IC $>0$. O coeficiente angular médio (omitindo-se quatro árvores em que a alta variância na forma dos frutos produziu um coeficiente angular inflacionado) foi 1,2015 (
0,3096; amplitude 0,2758-1,7942; $\mathrm{n}=34$ árvores); a mediana incluindo-se todas as árvores foi 1,207. Os coeficientes de $C$. aschersoniana indicam que a maior parte das árvores tem relação de dimensões variáveis em função do tamanho do fruto. Por se tratar de uma alometria positiva, o aumento em comprimento do fruto não está associado a um aumento correspondente do diâmetro, que neste caso é maior. Desta forma, um menor desenvolvimento em comprimento do que em diâmetro resulta em frutos mais achatados (oblatos) do que globosos (esféricos) ou alongados (prolatos).

Para todas as árvores de C. moschata (figura 1B), a linha de regressão foi: " $\ln ($ diâmetro de fruto) $=0,2509+$ $0,8789 \ln$ (comprimento de fruto)", com o coeficiente angular apresentando alometria negativa não significativa, ou isometria. Para cada árvore individualmente, oito das 22 apresentaram coeficiente angular $<1$, sendo que apenas três tiveram uma alometria negativa significativa. Das 14 árvores com coeficiente angular $>1$, três apresentaram alometria positiva significativa. $\mathrm{O}$ coeficiente angular médio (omitindo-se duas árvores com coeficientes inflacionados) foi 1,0416 ( 0,3046; amplitude 0,31511,$5570 ; \mathrm{n}=20$ ); o coeficiente angular mediano incluindose todas as árvores foi 1,0739. Com isto, de forma diversa ao encontrado aqui para os frutos de $C$. aschersoniana, tem-se que o aumento em comprimento do fruto de $C$. moschata apresentou-se com tendência a um aumento correspondente do diâmetro, resultando em frutos predominantemente esféricos a prolatos.

Para os diásporos de todas as árvores de $C$. aschersoniana (figura 1C), a linha de regressão foi dada pela equação: "ln(diâmetro) $=0,2333+1,2979$ ln(comprimento)", com $b$ indicando uma alometria positiva significativa. Em 13 das 36 árvores, o coeficiente angular foi $<1$, com apenas uma apresentando alometria negativa significativa. Das 23 árvores com coeficiente angular $>1$, apenas quatro apresentaram alometria positiva significativa. $\mathrm{O}$ coeficiente angular médio (omitindo-se cinco árvores com coeficientes inflacionados) foi 1,0971 ( 0,3414; amplitude 0,3199-1,8911; $\mathrm{n}=30$ ); o coeficiente angular mediano incluindo-se todas as árvores foi 1,1546.

A relação obtida para os diásporos de todas as árvores de C. moschata (figura 1D) foi dada pela equação: $" \ln ($ diâmetro $)=-1,1480+4,6380 \ln$ (comprimento)", com $b$ também apresentando alometria positiva significativa. Apenas duas das 22 árvores apresentaram coeficiente angular <1, com nenhuma apresentando alometria negativa significativa. Das 20 árvores com coeficiente angular $>1$, 10 apresentaram alometria positiva significativa. $\mathrm{O}$ coeficiente angular médio (omitindo-se cinco árvores com coeficientes inflacionados) foi 1,3108 ( 0,4503; amplitude $-0,2527-1,7989 ; \mathrm{n}=18)$; a mediana incluindo-se todas as árvores foi 1,4993 .

As diferenças na forma de frutos e diásporos das espécies estudadas, conforme a descrição apresentada por Moraes \& Paoli (1996), para C. moschata, podem ser causadas pela variação de seus tamanhos, como observado por Gould (1966), que aponta para a falha no entendimento 
da relação entre tamanho e forma como causa que tem levado os taxonomistas a enfatizar a variação na forma, quando, na realidade, é o tamanho que está variando. No entanto, Moraes \& Alves (1997) mostraram que para a espécie $C$. moschata, pela razão diâmetro/comprimento, tem-se que a maioria dos indivíduos da população do Parque Estadual Carlos Botelho apresentou frutos estritamente prolatos (16 indivíduos), enquanto que para os outros 11 indivíduos encontrou-se frutos prolatos, esféricos e oblatos, com apenas um destes indivíduos com frutos estritamente oblatos, mostrando que esta relação entre diâmetro e comprimento de frutos está associada à forma dos mesmos e reflete a variabilidade encontrada dentro da população. Adicionalmente, a análise de regressão do "eixo maior", para todas as árvores de $C$. moschata, também indicou uma tendência à isometria, ou seja, uma constância na forma tanto para frutos pequenos como para frutos grandes. No entanto, pela análise individualizada de todas as árvores, ficou evidenciado que podem ocorrer situações em que há uma maior elongação dos frutos com o aumento em tamanho (alometria negativa), ou seja, o comprimento do fruto aumenta a uma taxa maior que a do diâmetro, bem como pode haver um maior achatamento (alometria positiva), em que o diâmetro do fruto aumenta a uma taxa maior que a do comprimento. De forma semelhante, para os indivíduos de C. aschersoniana, tem-se que seis indivíduos apresentaram frutos inteiros estritamente prolatos, $20 \mathrm{com}$ frutos predominantemente prolatos, apenas um com frutos estritamente oblatos, e 10 com frutos predominantemente oblatos. Contudo, a análise de regressão do "eixo maior" para todas as árvores de $C$. aschersoniana mostrou uma tendência a uma alometria positiva, indicando a ocorrência de um maior achatamento dos frutos com o aumento em tamanho. Esses resultados discordam da tendência encontrada para a família Lauraceae de apresentar uma alometria negativa (elongação), conforme apontado por Mazer \& Wheelwright (1993). No entanto, esses autores sugerem a presença de alometria negativa em 16 das 20 espécies de Lauraceae de Monteverde, Costa Rica, apesar dos coeficientes angulares não terem sido significativamente diferentes de isometria, na maioria das espécies, o que significa dizer que é uma falsa tendência. Contudo, as espécies estudadas por esses autores na Costa Rica tinham seus diásporos dispersados por aves, enquanto que as duas espécies de Cryptocarya aqui tratadas são predominantemente dispersadas por primatas. Desta forma, a discordância da "tendência da família Lauraceae" pode ser decorrente apenas de adaptação diferenciada a vetores de dispersão distintos.

Para os diásporos, tem-se que apenas um indivíduo de $C$. moschata apresentou-os prolatos $\mathrm{e}$ esféricos, enquanto que todos os demais apresentaram diásporos estritamente prolatos. Apesar disto, a análise de regressão do "eixo maior" mostrou uma tendência dessa espécie produzir diásporos que aumentam mais rapidamente em diâmetro do que em comprimento com o tamanho (alometria positiva). Pela análise individualizada das árvores, a análise de regressão do "eixo maior" também mostrou a ausência de alometria negativa significativa. Para os indivíduos de $C$. aschersoniana, com exceção de dois que apresentaram diásporos prolatos e oblatos e um com diásporos prolatos e esféricos, todos os outros apresentaram diásporos estritamente prolatos. A análise de regressão do "eixo maior" para todas as árvores indicou uma tendência à alometria positiva, porém, a análise individualizada mostrou que a maior parte das árvores tendeu à isometria. Esses resultados também discordam da tendência encontrada por Mazer \& Wheelwright (1993), de alometria negativa para diásporos de espécies de Lauraceae.

Pela análise de agrupamento para as variáveis de frutos inteiros, observa-se a formação de três grupos de indivíduos (figura 2). O primeiro grupo é formado por 20 indivíduos, sendo 19 de $C$. moschata e apenas um de $C$. aschersoniana. Neste grupo, foram englobados aqueles indivíduos que apresentaram frutos pequenos, com médias de dimensões variando entre 1,93 e $2,60 \mathrm{~cm}$ de comprimento, 1,52 e 2,24 cm de diâmetro, 9,72 e $15,04 \mathrm{~cm}^{2}$ de superfície, e 2,76 e $5,52 \mathrm{~cm}^{3}$ de volume. Com exceção dos indivíduos CB19 e BR01, cujos frutos eram predominantemente oblatos, todos os demais possuíam frutos predominantemente prolatos. Desta forma, apreende-se que este agrupamento englobou quase a totalidade dos indivíduos de C. moschata, cujos frutos são relativamente menores, e o indivíduo de $C$. aschersoniana que possuía frutos com dimensões semelhantes aos da primeira, e que foi coletado na Fazenda Barreiro Rico em área denominada por "cerrado". O segundo agrupamento englobou 31 indivíduos, sendo 28 de C. aschersoniana e apenas três de $C$. moschata, em que os frutos eram de tamanho intermediário, variando com médias entre 2,18 e $2,73 \mathrm{~cm}$ de comprimento, 2,11 e 2,58 cm de diâmetro, 15,46 e $20,39 \mathrm{~cm}^{2}$ de superfície, e 5,70 e $8,85 \mathrm{~cm}^{3}$ de volume. Por sua vez, o terceiro agrupamento reuniu os oito indivíduos de $C$. aschersoniana que possuíam em média as maiores dimensões, variando entre 2,61 e 2,99 cm de comprimento, 2,50 e 2,69 cm de diâmetro, 21,75 e 24,45 $\mathrm{cm}^{2}$ de superfície, e 9,42 e $11,16 \mathrm{~cm}^{3}$ de volume. Com isto, tem-se mais uma vez que a ausência de uma nítida descontinuidade, ou seja, de um intervalo entre as distribuições dos valores de uma variável das duas espécies, fez com que os grupos 1 e 2 apresentassem elementos de ambas.

De forma semelhante, a análise de agrupamento das medidas de diásporos também formou três grupos, apresentando adicionalmente um indivíduo que não se agrupou (figura 3). O primeiro grupo é formado por 32 indivíduos, sendo 18 de $C$. aschersoniana e 14 de $C$. moschata, englobando aqueles com dimensões que variaram em média entre 1,91 e $2,54 \mathrm{~cm}$ de comprimento, 1,38 e $1,63 \mathrm{~cm}$ de diâmetro, 8,85 e $9,93 \mathrm{~cm}^{2}$ de superfície, e 2,36 e $2,79 \mathrm{~cm}^{3}$ de volume. O segundo grupo, composto por 11 indivíduos de C. aschersoniana e quatro de C. moschata, abrangeu aqueles com variação em termos médios entre 1,81 e $2,19 \mathrm{~cm}$ de comprimento, 1,29 e $1,55 \mathrm{~cm}$ de diâmetro, 7,27 e $8,67 \mathrm{~cm}^{2}$ de superfície, e 1,76 e $2,33 \mathrm{~cm}^{3} \mathrm{de}$ volume, contendo, desta forma, diásporos menores que os 
do grupo anterior. O terceiro grupo foi formado por seis indivíduos de C. aschersoniana e quatro de C. moschata, os quais apresentaram os diásporos com grandes dimensões, variando em média entre 2,07 e 2,45 cm de comprimento, 1,53 e 1,84 cm de diâmetro, 10,20 e $11,62 \mathrm{~cm}^{2}$ de superfície, e 2,96 e $3,72 \mathrm{~cm}^{3}$ de volume. $\mathrm{O}$ indivíduo não agrupado apresentou diásporos com médias de $2,56 \mathrm{~cm}$ de comprimento, $1,86 \mathrm{~cm}$ de diâmetro, $14,22 \mathrm{~cm}^{2}$ de superfície, e $4,71 \mathrm{~cm}^{3}$ de volume.

As análises de agrupamento para as variáveis de frutos inteiros e de diásporos estabeleceram uma separação dos indivíduos das espécies de Cryptocarya em função da dimensão de seus frutos e diásporos. Com isto, distinguiram-se três agrupamentos de indivíduos, para frutos inteiros, com frutos pequenos, de tamanho intermediário, e com frutos relativamente grandes, respectivamente. Da mesma forma, para os diásporos, os três agrupamentos formados também foram estabelecidos a partir de uma gradação de indivíduos com diásporos pequenos, intermediários e grandes. A análise de agrupamento dos indivíduos para os frutos inteiros mostrou haver uma maior homogeneidade dentro dos grupos formados por cada espécie, do que o apresentado pela análise de agrupamento para os diásporos, o que já havia sido indicado pelas análises de regressão logística e de função linear discriminante. Observa-se uma grande variação na relação entre as dimensões de frutos inteiros dos indivíduos com as respectivas dimensões de seus diásporos. Desta forma, dos 20 indivíduos do primeiro agrupamento (frutos pequenos), quatro apresentaram diásporos pequenos, 13 com diásporos intermediários, e três com diásporos grandes. Dos 31 indivíduos do segundo agrupamento (frutos intermediários), 10 apresentaram diásporos pequenos, 15 com diásporos intermediários, e cinco com diásporos grandes. Dos oito indivíduos do terceiro agrupamento (frutos grandes), nenhum apresentou diásporos pequenos, quatro apresentaram diásporos intermediários, dois com diásporos grandes, e um com diásporos muito grandes. Com isto, tem-se que os frutos inteiros de C. moschata, da população de Carlos Botelho $\mathrm{SP}$, são menores, em sua grande maioria, que os de $C$. aschersoniana do interior do mesmo estado e sul de Minas Gerais. No entanto, esta predominância de frutos pequenos em $C$. moschata não está necessária e diretamente associada a uma relação recíproca de produção de diásporos também pequenos, sendo que a maioria de seus indivíduos apresentaram diásporos com dimensões intermediárias. Da mesma forma, os frutos inteiros e predominantemente maiores de $C$. aschersoniana também apresentaram variação quanto ao tamanho de seus diásporos, havendo uma predominância de indivíduos que produziram frutos intermediários com diásporos intermediários. Adicionalmente, o fato de se obter agrupamentos distintos e de os mesmos não serem formados em função das localizações desses indivíduos, indica que a variação encontrada nas dimensões de seus frutos e diásporos não é promovida apenas por fatores ambientais, mas também por variabilidade genética populacional. Esses resultados são semelhantes aos reportados por Sano et al. (1999), para frutos e sementes de Dipteryx alata Vog.

Cabe ressaltar que o tamanho de alguns frutos de C. aschersoniana amostrados aqui foi maior do que o descrito na literatura. Isto pode ser explicado pela amostragem deste trabalho ter abrangido uma maior amplitude de variação dentro das espécies estudadas do que aquelas realizadas apenas com os materiais disponíveis nos herbários, que por sua vez são bastante escassos, apesar de referirem-se a uma representatividade geográfica das espécies muito maior do que a amostragem realizada no presente estudo. Adicionalmente, os espécimens de herbário, por perda de água, têm o seu tamanho e forma alterados, o que geralmente se dá por uma diminuição de suas proporções, além do fato de muitos serem coletas de indivíduos com frutos ainda imaturos, o que nem sempre é mencionado por seus coletores. Desta forma, as mensurações apresentadas aqui para a amostragem realizada em $C$. aschersoniana revelaram dimensões de diâmetros principal e consideravelmente superiores aos registrados na literatura. Outro fator a ser considerado refere-se ao fato de que dentro do binômio $C$. aschersoniana Mez existem pelo menos três diferentes morfoespécies cujos status taxonômicos permanecem duvidosos quanto a se realmente representam uma única espécie biológica. Desta forma, tem-se que os indivíduos amostrados no presente trabalho pertencem todos ao mesmo padrão morfológico encontrado nas populações do Planalto de São Paulo e sul de Minas Gerais, semelhante àquele apresentado pela coleta de Sellow s.n. (B+, duplicatas e/ou fragmentos em E, F, K, KIEL, L, LE, NY e US), localidade não indicada, que é um dos sintipos de $C$. moschata Nees, mas que na revisão feita por Kostermans (1937, 1938) foi sinonimizado em C. aschersoniana Mez. Por outro lado, indivíduos provenientes principalmente do sul do Brasil, com o padrão típico de $C$. aschersoniana senso Mez (1889), holótipo Sellow s.n., local não indicado $(\mathrm{B}+$, fragmento em $\mathrm{F})$, apresentam frutos e diásporos notoriamente menores que os do padrão anterior, sendo inclusive menores que os de $C$. moschata, com diásporos majoritariamente sem costulação alguma.

A importância de se ressaltar esta ocorrência deve-se ao fato de que as dimensões dos frutos, especialmente o diâmetro, têm mostrado uma associação significativa com o tipo de dispersor, tendendo a aumentar com o aumento da participação de mamíferos entre os agentes dispersores (Jordano 1995). O gênero Cryptocarya está entre aqueles que demonstram uma tendência para um aumento em tamanho médio de fruto, a partir de congenéricos dispersados por aves, com valores intermediários para táxons com dispersão mista (aves e mamíferos), culminando com os de dispersão por mamíferos (Jordano 1995). As duas espécies estudadas aqui apresentam frutos e diásporos grandes, reduzindo o número de dispersores de seus diásporos (P.L.R Moraes, dados não publicados). Tanto $C$. aschersoniana como $C$. moschata possuem frutos dispersados pelo Brachyteles arachnoides (Primates, Cebidae) [Moraes \& Paoli, 1995, C.T. Assumpção, dados não publicados], assim como por 
Alouatta fusca (Kuhlmann 1975, Galetti et al. 1994).

Adicionalmente, o tamanho de diásporos de espécies arbóreas tropicais é uma característica da história vital que pode afetar o valor adaptativo das árvores-mãe e do processo de regeneração da população. Tem-se observado que os diásporos grandes aumentam o sucesso germinativo, o crescimento e sobrevivência da plântula, como consequiência da produção de plântulas mais vigorosas e competitivamente superiores. Como exemplo, tem-se um estudo realizado com Cryptocarya alba (Mol.) Looser, onde encontrou-se que o tamanho da plântula estava altamente relacionado com o tamanho do diásporo, sendo que a alocação de biomassa nas raízes foi proporcionalmente maior em plântulas oriundas de diásporos pequenos, enquanto que a alocação em folhas foi maior naquelas oriundas de diásporos grandes (Chacon et al. 1998). Sob condições particulares, a seleção pode favorecer a evolução de diásporos grandes em detrimento dos custos potenciais em se reduzir sua dispersão. Por exemplo, os diásporos grandes podem ser necessários para o estabelecimento bem sucedido de plântulas sob condições de baixa luminosidade (Mazer \& Wheelwright 1993), fato este observado para C. moschata (Moraes \& Paoli 1999).

\section{Agradecimentos}

Ao Instituto Florestal de São Paulo, Instituto de Botânica, Prefeitura Municipal de Campinas e a José Carlos Reis de Magalhães, pela autorização de coleta de material.

\section{Referências bibliográficas}

BERNARDI, L. 1962. Lauraceas. Universidad de Los Andes, Facultad de Ciencias Forestales, Talleres Graficos Universitarios, Merida.

CHACON, P., BUSTAMANTE, R. \& CAROLINA, H. 1998. The effect of seed size on germination and seedling growth of Cryptocarya alba (Lauraceae) in Chile. Rev. Chilena Hist. Natur. 71:189-197.

COE-TEIXEIRA, B. 1965. Lauráceas do Estado de São Paulo - II: Cryptocarya. Arq. Bot. São Paulo 4:3-8.

GALETTI, M., PEDRONI, F. \& MORELATTO, L.P.C. 1994. Diet of the brown howler monkey Alouatta fusca in a forest fragment in southeastern Brazil. Mammalia 58:111-118.

GOULD, S.J. 1966. Allometry and size in ontogeny and phylogeny. Biol. Rev. 41:587-640.

HOWARD, R.A. 1981. Nomenclatural notes on the Lauraceae of the Lesser Antilles. J. Arnold Arbor. 62:45-62.

JOLICOUER, P. \& HEUSNER, A.A. 1971. The allometry equation in the analysis of the standard oxygen consumption and body weight of the white rat. Biometrics 27:841-855.

JOLY, C.A., AIDAR, M.P.M., KLINK, C.A., McGRATH, D.G., MOREIRA, A. G., MOUTINHO, P., NEPSTAD, D.C., OLIVEIRA, A.A., POTT, A., RODAL, M.J.N. \& SAMPAIO, E.V.S.B. 1999.
Evolution of the Brazilian phytogeography classification systems: implications for biodiversity conservation. Ciência e Cultura 51:331-348.

(Http://www.unicamp.br/ib/botanica/CCultura1.htm)

JORDANO, P. 1995. Angiosperm fleshy fruits and seed dispersers: a comparative analysis of adaptation and constraints in plant-animal interactions. Am. Nat. 145:163-191

KOSTERMANS, A.J.G.H. 1937. Revision of the Lauraceae II. The genera Endlicheria, Cryptocarya (american species) and Licaria. Med. Bot. Mus. Herb. Rijk. Univ. Utrecht 42:500-609.

KOSTERMANS, A.J.G.H. 1938. Revision of the Lauraceae III. The genera Aiouea, Systemonodaphne, Urbanodendron, Mezilaurus; additions and corrections to Licaria and Cryptocarya. Recueil. Trav. Bot. Néerl. 35:56-129.

KUHLMANN, M. 1975. Adenda alimentar dos bugios. Silvic. S. Paulo 9:57-62.

LABARBERA, M. 1989. Analyzing body size as a factor in ecology and evolution. Ann. Rev. Ecol. Syst. 20:97117.

MAZER, S.J. \& WHEELWRIGHT, N.T. 1993. Fruit size and shape: allometry at different taxonomic levels in bird-dispersed plants. Evol. Ecol. 7:556-575.

MEZ, C. 1889. Lauraceae americanae. Jahrb. Königl. Bot. Gart. Berlin 5:1-556.

MORAES, P.L.R. \& ALVES, M.C. 1997. Biometria de frutos e sementes de Cryptocarya moschata Nees, Ocotea catharinensis Mez e Endlicheria paniculata (Sprengel) MacBride (Lauraceae). Bol. Mus. Biol. Mello Leitão (N. Sér.) 6:23-34.

MORAES, P.L.R. \& PAOLI, A.A.S. 1995. Dispersão e germinação de sementes de Cryptocarya moschata Nees \& Martius ex Nees, Ocotea catharinensis Mez e Endlicheria paniculata (Sprengel) MacBride (Lauraceae). Arq. Biol. Tecnol. 38:1119-1129.

MORAES, P.L.R. \& PAOLI, A.A.S. 1996. Morfologia de frutos e sementes de Cryptocarya moschata Nees \& Martius ex Nees, Endlicheria paniculata (Sprengel) MacBride, e Ocotea catharinensis Mez (Lauraceae). Rev. Brasil. Sementes 18:17-27.

MORAES, P.L.R. \& PAOLI, A.A.S. 1999. Morfologia e estabelecimento de plântulas de Cryptocarya moschata Nees, Ocotea catharinensis Mez e Endlicheria paniculata (Spreng.) MacBride Lauraceae. Rev. Bras. Bot. 22(Supl.2):287-295. (http://www.scielo.br/scielo.php?script=sci arttext\& pid=S0100-84041999000500010\&lng=en\&nrm=iso)

RICHTER, H.G. 1981. Anatomie des sekundären Xylems und der Rinde der Lauraceae. Sonderb. Naturwiss. Vereins, Hamburg 5:1-148.

ROHWER, J.G. 1993a. Lauraceae: Nectandra. Flora Neotropica 60:1-332.

ROHWER, J.G. 1993b. Lauraceae. In The Families and Genera of Vascular Plants, v.2. (K. Kubitzki, J.G. Rohwer \& V. Bittrich, eds.). Springer-Verlag, Berlin, p.366-391. 
SANO, S.M., VIVALDI, L.J. \& SPEHAR, C.R. 1999. Diversidade morfológica de frutos e sementes de baru (Dipteryx alata Vog.). Pesq. Agropec. Bras. 34:513518 .

SAS INSTITUTE. 1989. SAS/STAT ${ }^{\circledR}$ user's guide. Version 6, 4th. ed., v. 1. SAS Institute Inc., Cary, N.C.

SOKAL, R.R. \& ROHLF, F.J. 1995. Biometry. 3ed. W.H. Freeman \& Co., New York.

TURRELL, F.M. 1946. Tables of Surfaces and Volumes of Spheres and of Prolate and Oblate Spheroids, and Spheroidal Coefficients. University of California Press, Berkeley.

VAN DER WERFF, H. 1988. Eight new species and one new combination of neotropical Lauraceae. Ann. MO. Bot. Garden 75:402-419.

VAN DER WERFF, H. 1991. A key to the genera of Lauraceae in the New World. Ann. MO. Bot. Garden 78:377-387.

VATTIMO-GIL, I. 1966. Notas sôbre o gênero Cryptocarya R. BR. no Brasil (Lauraceae). Rodriguésia 37:219-237.

VATTIMO-GIL, I. 1979. Lauráceas. Flora Ilustrada Catarinense, Itajaí, SC.
Título: Biometria de frutos e diásporos de Cryptocarya aschersoniana Mez e Cryptocarya moschata Nees (Lauraceae)

Autor: Pedro Luís Rodrigues de Moraes e Marcelo Corrêa Alves

Biota Neotropica, Volume 2, número 1, 2002 -

http://www.biotaneotropica.org.br/v2n1/pt/abstract?articl e+BN01302012002

Recebido em 17 de janeiro de 2002

Revisado em 26 de março de 2002

Aceito em 29 de abril de 2002

ISSN 1676-0603 
Ciryptocarya aschersomiana

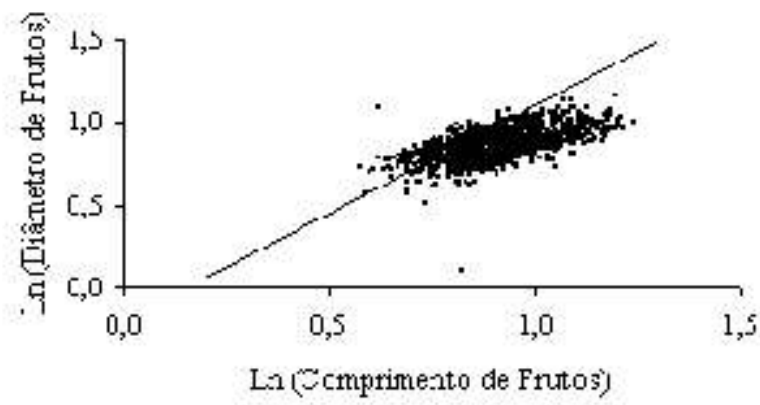

Fig. 1A

\section{Cryptocarya aschersoniana}

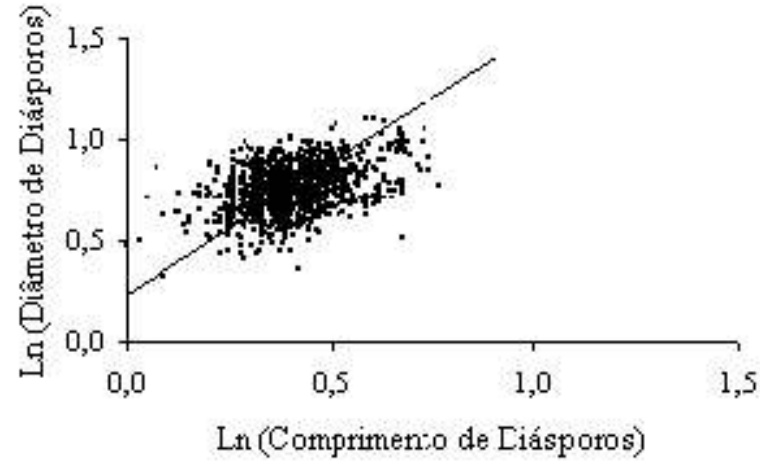

Fig. 1C

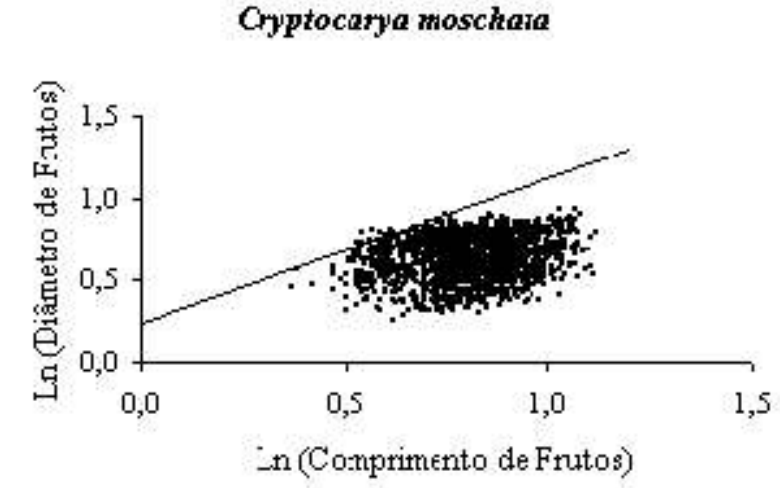

Fig. 1B ig. $1 \mathrm{~B}$

\section{Gyptocarya moschata}

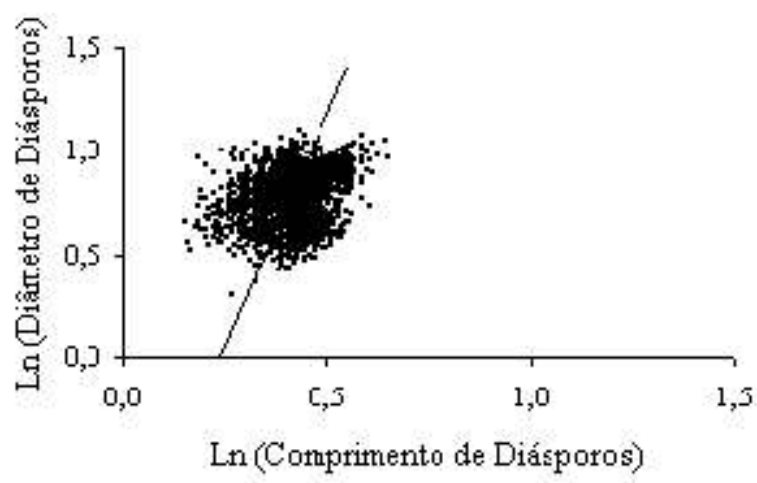

Fig. 1D

Figuras 1A-D. A. Relação entre o $\ln ($ comprimento de fruto) e $\ln$ (diâmetro de fruto) de todas as árvores de Cryptocarya aschersoniana Mez; o coeficente angular da regressão do "eixo maior" foi 1,30. B. Relação entre o ln(comprimento de fruto) e ln(diâmetro de fruto) de todas as árvores de Cryptocarya moschata Nees; o coeficiente angular da regressão do "eixo maior" foi 0,87 . C. Relação entre o $\ln$ (comprimento de diásporo) e $\ln$ (diâmetro de diásporo) de todas as árvores de Cryptocarya aschersoniana Mez; o coeficente angular da regressão do "eixo maior" foi 1,29. D. Relação entre o $\ln$ (comprimento de diásporo) e ln(diâmetro de diásporo) de todas as árvores de Cryptocarya moschata Nees; o coeficiente angular da regressão do "eixo maior" foi 4,63. 


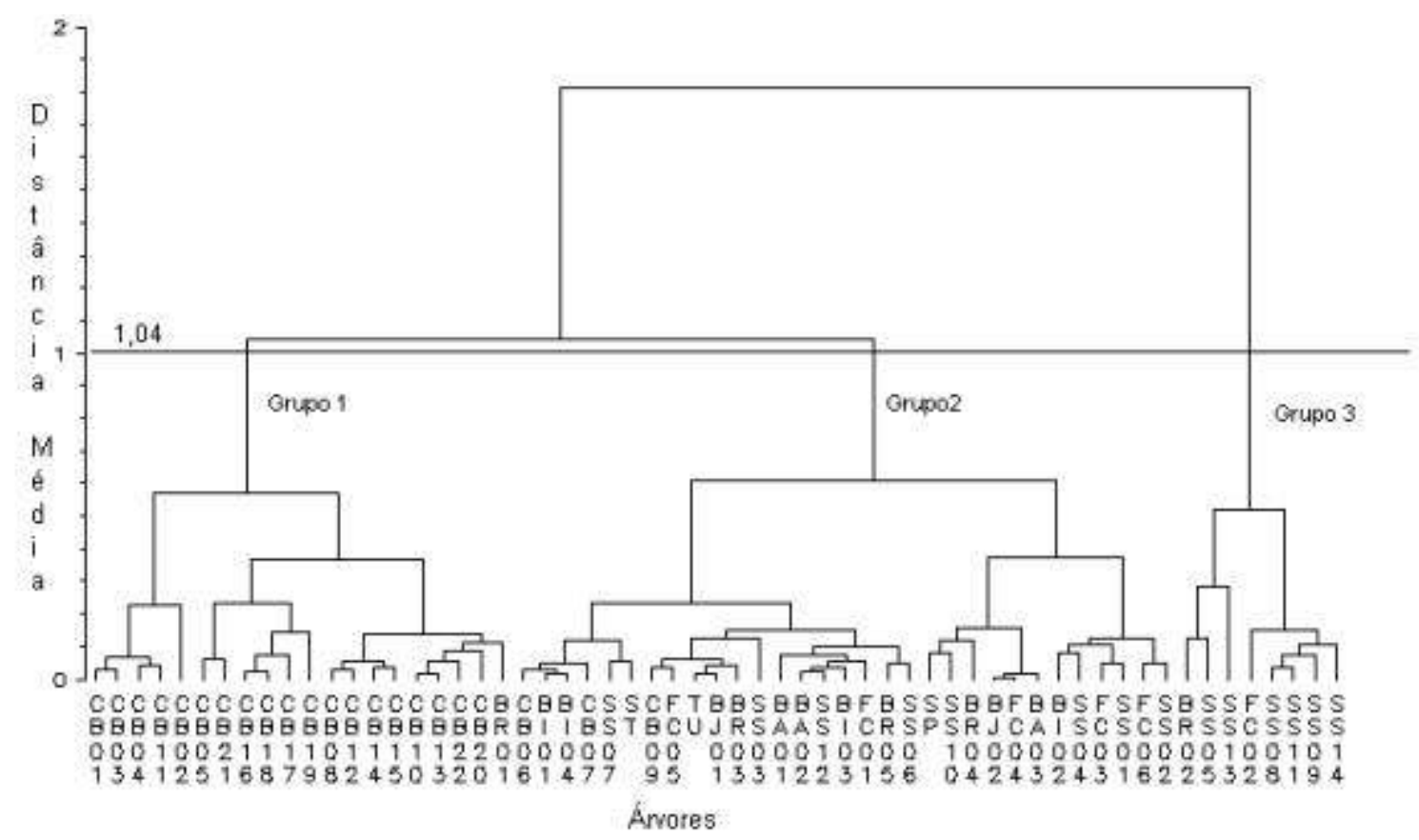

Figura 2. Análise de agrupamento para as variáveis de frutos inteiros de árvores de Cryptocarya aschersoniana Mez e $C$. moschata Nees, a partir de distância Euclidiana. Método de ligação média. Ponto de corte determinado pela estatística pseudo- $\mathrm{t}^{2}=1,04$. Código das árvores conforme tabela 1 .

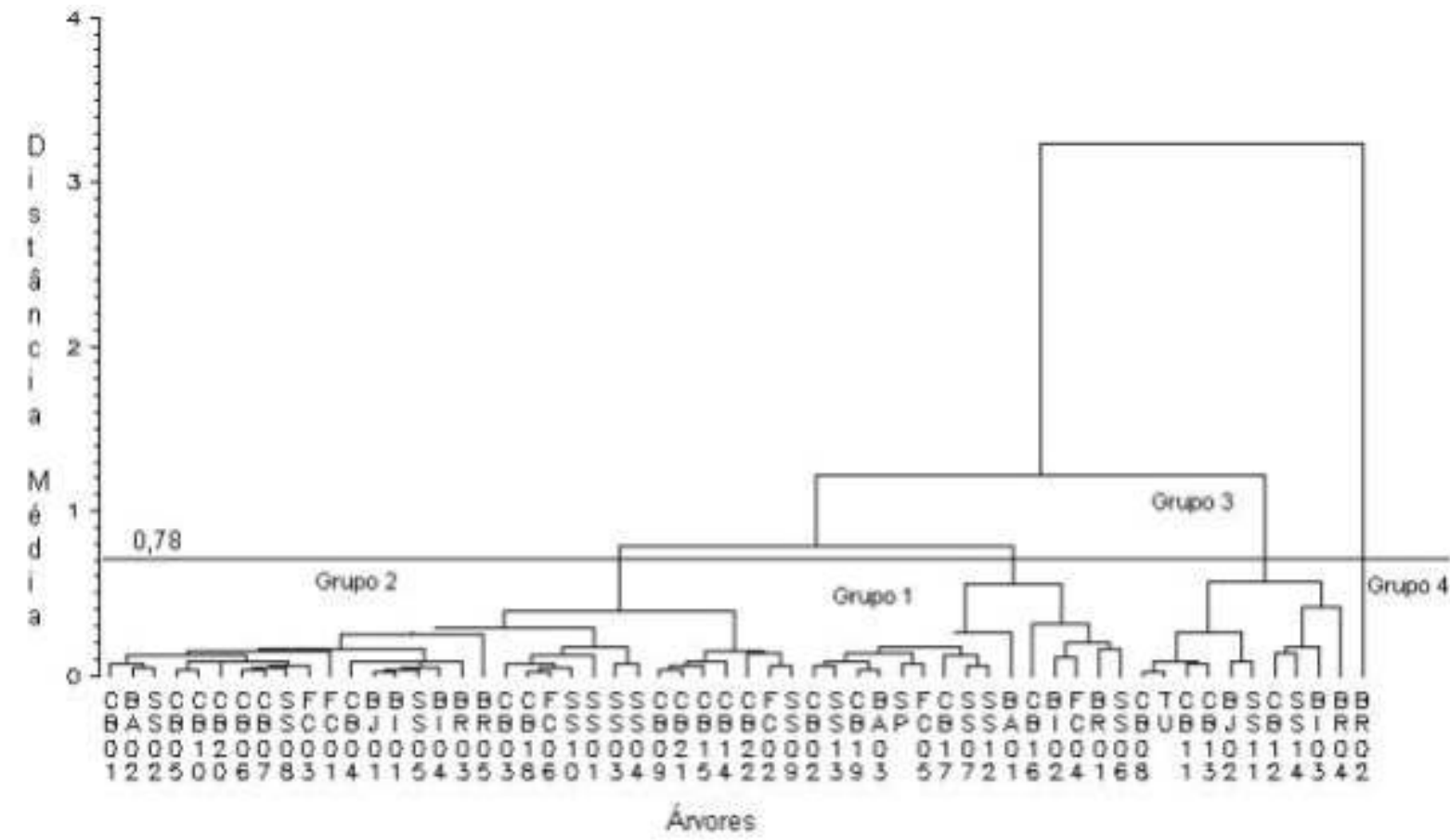

Figura 3. Análise de agrupamento para as variáveis de diásporos de árvores de Cryptocarya aschersoniana Mez e $C$. moschata Nees, a partir de distância Euclidiana. Método de ligação média. Ponto de corte determinado pela estatística pseudo- $\mathrm{t}^{2}=0,78$. Código das árvores conforme tabela 1 . 
Tabela 1: Áreas de coleta de frutos de Cryptocarya spp. com os respectivos números de matrizes e de frutos e diásporos analisados. Código das áreas entre parênteses.

\begin{tabular}{|c|c|c|c|c|c|}
\hline Espécie & Área & Município & $\begin{array}{c}\mathrm{N}^{\mathrm{o}} \text { de } \\
\text { matrizes }\end{array}$ & $\mathrm{N}^{\mathrm{o}}$ de frutos & $\begin{array}{c}\mathrm{N}^{\mathrm{o}} \text { de } \\
\text { diásporos }\end{array}$ \\
\hline C. moschata & $\begin{array}{l}\text { Parque Estadual Carlos Botelho } \\
\text { (CB); 24S 03'54', 47W 57'54" }\end{array}$ & São Miguel Arcanjo & 22 & 1.892 & 1.764 \\
\hline C. aschersoniana & $\begin{array}{l}\text { Horto Florestal de Tupi (TU); } \\
\text { 22S 43'44”, 47W 31'15" }\end{array}$ & Piracicaba & 1 & 54 & 54 \\
\hline C. aschersoniana & $\begin{array}{l}\text { Serra de São Pedro (SP); } \\
\text { 22S 29’53”, 47W 54’53” }\end{array}$ & São Pedro & 1 & 54 & 54 \\
\hline C. aschersoniana & $\begin{array}{l}\text { Bosque dos Jequitibás (BJ); } \\
\text { 22S 54'23", 47W 03'06" }\end{array}$ & Campinas & 2 & 72 & 54 \\
\hline C. aschersoniana & $\begin{array}{l}\text { Bosque dos Alemães (BA); } \\
\text { 22S 53'24”, 47W 04'07'” }\end{array}$ & Campinas & 3 & 156 & 156 \\
\hline C. aschersoniana & $\begin{array}{l}\text { Bosque dos Italianos (BI); } \\
\text { 22S 53'14”, 47W 04'23”' }\end{array}$ & Campinas & 4 & 216 & 216 \\
\hline C. aschersoniana & $\begin{array}{l}\text { Fazenda Barreiro Rico (BR); } \\
\text { 22S 40'06”, 48W 08'23"' }\end{array}$ & Anhembi & 5 & 266 & 189 \\
\hline C. aschersoniana & $\begin{array}{l}\text { Fazenda Campininha (FC); } \\
\text { 22S 14'46”, 47W 10’21”' }\end{array}$ & Mogi Guaçu & 6 & 312 & 168 \\
\hline C. aschersoniana & $\begin{array}{l}\text { Sul de Minas Gerais (SS e ST); } \\
\text { 21S 01'24", 47W 00'12", e } \\
\text { 20S 49'46", 47W 03'09"' }\end{array}$ & $\begin{array}{c}\text { São Sebastião do } \\
\text { Paraíso e São Tomás de } \\
\text { Aquino }\end{array}$ & 15 & 357 & 392 \\
\hline
\end{tabular}

Tabela 2: Estatística descritiva para as espécies Cryptocarya aschersoniana $\mathrm{Mez}$ (frutos, $\mathrm{N}=1.487$; diásporos, $\mathrm{N}=1.283$ ) e C. moschata $\mathrm{Nees}$ (frutos, $\mathrm{N}=1.892$; diásporos, $\mathrm{N}=1.764$ ), com respectivos testes $t$ para as médias.

\begin{tabular}{lccccccccc}
\hline & \multicolumn{4}{c}{ C. aschersoniana } & \multicolumn{3}{c}{ C. moschata } \\
\hline Variável & Média & Mínimo & Máximo & $\begin{array}{c}\text { Desvio } \\
\text { Padrão }\end{array}$ & Média & Mínimo & $\begin{array}{c}\text { Máximo } \\
\text { Desvio } \\
\text { Padrão }\end{array}$ & $\begin{array}{c}\text { Teste } \\
t\end{array}$ \\
\hline Comprimento de fruto $(\mathrm{cm})$ & 2,50 & 1,78 & 3,47 & 0,28 & 2,26 & 1,45 & 3,06 & 0,28 & $* *$ \\
Diâmetro de fruto $(\mathrm{cm})$ & 2,37 & 1,10 & 3,20 & 0,22 & 1,90 & 1,29 & 2,55 & 0,24 & $* *$ \\
Diâmetro:comprimento & 0,95 & 0,48 & 1,61 & 0,08 & 0,85 & 0,54 & 1,27 & 0,14 & $* *$ \\
Superfície de fruto $\left(\mathrm{cm}^{2}\right)$ & 18,33 & 6,95 & 32,96 & 3,45 & 12,91 & 6,71 & 22,32 & 2,61 & $* *$ \\
Volume de fruto $\left(\mathrm{cm}^{3}\right)$ & 7,56 & 1,44 & 17,69 & 2,06 & 4,32 & 1,62 & 9,87 & 1,34 & $* *$ \\
Comprimento de diásporo $(\mathrm{cm})$ & 2,15 & 1,38 & 3,00 & 0,24 & 2,17 & 1,34 & 3,00 & 0,27 & n.s. \\
Diâmetro de diásporo $(\mathrm{cm})$ & 1,50 & 1,00 & 2,16 & 0,15 & 1,52 & 1,16 & 1,92 & 0,11 & n.s. \\
Diâmetro:comprimento & 0,70 & 0,46 & 1,17 & 0,08 & 0,71 & 0,45 & 0,98 & 0,09 & n.s. \\
Superfície de diásporo $\left(\mathrm{cm}^{2}\right)$ & 9,43 & 4,35 & 17,83 & 1,83 & 9,43 & 5,47 & 15,35 & 1,41 & n.s. \\
Volume de diásporo $\left(\mathrm{cm}^{3}\right)$ & 2,61 & 0,84 & 6,50 & 0,75 & 2,65 & 1,20 & 5,42 & 0,58 & n.s. \\
\hline
\end{tabular}

** $\mathrm{P}<0,01 ;$ n.s.: não significativo

Tabela 3: Número de observações de frutos e diásporos, com as respectivas porcentagens, classificadas de espécie para espécie.

\begin{tabular}{lllllll}
\hline \multicolumn{1}{c}{ Espécie } & \multicolumn{2}{c}{ C. aschersoniana } & \multicolumn{2}{c}{ C. moschata } & \multicolumn{2}{c}{ Total } \\
\hline \multirow{3}{*}{ C. aschersoniana } & Frutos & Diásporos & Frutos & Diásporos & Frutos & Diásporos \\
& 1.229 & 789 & 258 & 494 & 1.487 & 1.283 \\
C. moschata & $82,65 \%$ & $61,50 \%$ & $17,35 \%$ & $38,50 \%$ & $100 \%$ & $100 \%$ \\
& 243 & 402 & 1.649 & 1.362 & 1.892 & 1.764 \\
\hline Total & $12,84 \%$ & $22,80 \%$ & $87,16 \%$ & $77,20 \%$ & $100 \%$ & $100 \%$ \\
Porcentagem & 1.472 & 1.191 & 1.907 & 1.856 & 3.379 & 3.047 \\
Esperado & 43,56 & 39,09 & 56,44 & 60,91 & 100 & 100 \\
\hline
\end{tabular}

